\title{
Early strength development of shotcrete for rapid mine development and behaviour under dynamic loads
}

\author{
F Erismann Sika, Switzerland \\ M Hansson Sika, Sweden
}

\begin{abstract}
Ever deeper mine developments combined with wider spans and layouts with close infrastructure alignments represent an increasing challenge for installed underground support. Destressing and sudden seismic events frequently create severe damage to the vital underground support within the haulage, ore handling and extraction infrastructure. In-cycle shotcrete has proven to be the primary support of choice in most large-scale mine developments, including the recent super-cave developments, such as Oyu Tolgoi, Chuquicamata, El Teniente and Grasberg. As a result, increased efforts have been undertaken over the past years to understand the behaviour of fibre-reinforced sprayed concrete under sudden dynamic loads, with evaluations of the latest developments in fibre technologies and shotcrete mix designs. Together with Luossavaara-Kiirunavaara Aktiebolag (LKAB) and the operating team at the Kiirunavaara and Malmberget operations in Sweden, among the world's most stringent operations when it comes to early strength requirements and energy absorption of the shotcrete liner, Sika undertook extensive testing of different shotcrete mix designs. Simulated seismic shocks were used in order to evaluate primary shotcrete liners by observing their behaviour under such rapid deformation events (Swedberg 2013; Swedberg et al. 2014). The major findings and test results form part of this paper. Particular focus was also given to the early strength development of the sprayed shotcrete because destressing events usually occur soon after a face has been blasted (Amberg 2006), and thus rapid strength development is of particular interest. For reliable in situ onsite strength development measurement, Sika developed an ultrasonic-based tool to measure the strength of the sprayed concrete at the sprayed location and will allow development teams to re-enter secured perimeters at the right time. This is seen to have a major positive impact on safety and productivity of any rapid mine development project.
\end{abstract}

Keywords: shotcrete, concrete, fibre, accelerator, early strength, cycle time, blast cycle, efficiency, ultrasound, MiniShot, Sika, LKAB, block cave, sprayed concrete, yielding, energy absorption, seismic, destressing, mining, underground, rapid development

\section{Introduction}

Due to its involvement at many mine sites globally, Sika was able to gather a large database by testing different primary underground support designs over the years. The collaboration with LuossavaaraKiirunavaara Aktiebolag (LKAB) in Sweden was particularly close, and a substantial amount of the data compiled in this study originates from the LKAB Kiirunavaara and Malmberget mine sites. Data from other sites were also included. The goal of the study was to create an overview of the energy absorption capacity of the different support systems and their combinations as well as the amount of energy absorbed and deflection created during sudden impact events. The breaking pattern in relation to impact energy was another critical factor to observe. Most of the current data supports what is already implemented at many mine sites. Today, fibre-reinforced shotcrete is the go-to solution at many mine sites because it can be implemented in an efficient in-cycle schedule. However, meshing is still widely used, either in combination with shotcrete or without, due to its extensive yielding component, which provides a passive support system, and its good energy absorption capacity in combination with sprayed concrete. Fibre-reinforced shotcrete in combination with mesh has proven to be a resilient support when it comes to blast damage, 
seismic shocks and destressing events. This combination of systems also maintains the support integrity, with a view towards energy absorption as well as long-term stability in a corrosive environment. Coupled with a timely and cost-effective installation, such support designs can be considered the best choice for deep-level mines and large-scale deep-footprint developments.

\section{Methodology}

Many underground support methods have been discussed and evaluated over the past decades. Several dynamic testing rigs have been installed over the years at different institutions (Hadjigeorgiou \& Potvin 2011). However, standardised testing of the various systems-and hence comparability of data-has been largely neglected, and there is a lack of data harmonisation among the various testing rigs. For this study, two large data sets have been used and compiled. First, standardised testing results according to the ASTM C1550 standard (ASTM International 2012) have been used, focusing on the major in-cycle support installations. Second, results from dynamic impact testing have been compiled in order to link results to the more standardised ASTM round panel results. Fibre-reinforced shotcrete, using steel fibres of different type and dosage or polypropylene fibres of different type and dosage are today an integral part of modern mine developments because they allow for rapid mine cycles and active ground support early on after spraying, good quality-control measures and good scalability of the installed support. Hence, shotcrete is the main focus of this paper. The use of mesh or screens remains common at many mine sites. This is mainly due to the large deflection and good energy absorption that can be achieved, coupled with restricted access for equipment and concrete that would be used for an alternative sprayed concrete support. As installation of bolts and meshes remains the primary support installation of choice at a number of mines, welded meshes were included in the evaluation process, either as a standalone support or in combination with shotcrete. Many studies have already evaluated different shotcrete mixes. However, only a few have placed adequate emphasis on the comparability of data. The effectiveness of a good shotcrete liner greatly depends on the quality of the produced concrete to be sprayed, the interaction with the accelerator chemistry, the quality and amount of fibres and the application of the sprayed concrete. These aspects all leave a lot of room for data scattering when analysing actual sprayed samples. In order to avoid this bias, it was decided that casted ASTM panel samples would be evaluated. As shown in Figure 1a, round panels with thicknesses ranging from 50 to $100 \mathrm{~mm}$ and diameters of $800 \mathrm{~mm}$ were tested according to the ASTM standard C 1550-04, where flexural toughness of the reinforced panels is evaluated through central loading of the panel until a deflection of $40 \mathrm{~mm}$ is reached. As shown in Figure 1b, dynamic impact testing was evaluated at the LKAB mine site using a drop-testing rig to simulate impact energies of different magnitudes and the effects they would have on the reinforced panel or mesh. For this study, only those values where the panel remained intact after the impact were used (Swedberg \& Krutrök 2016).

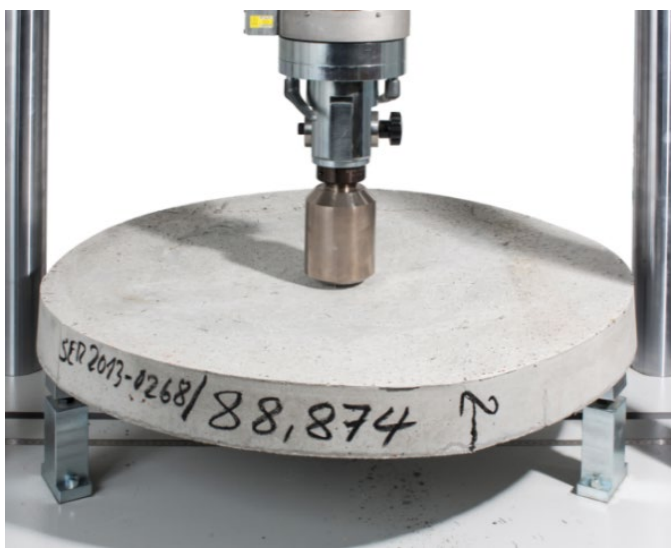

(a)

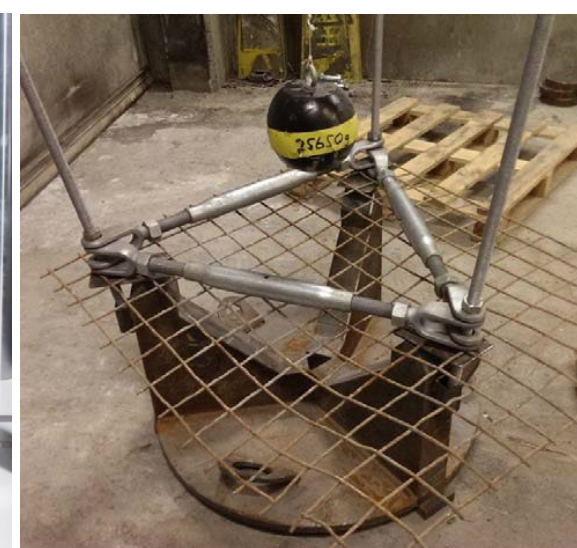

(b)

Figure 1 (a) ASTM round panel test rig; (b) LKAB dynamic impact test rig with a mounted, welded wire mesh and drop weight 


\section{Database}

Results from well over 200 ASTM panels were the foundation of this study. Most data were gathered from QAQC testing data of mine sites and dynamic impact data that were gathered for this study at the LKAB QC facility in Kiruna, Sweden (Thyni 2014). The following support designs were tested under standardised ASTM C1550 protocols:

- Fibre-reinforced shotcrete using polypropylene macro fibres from various suppliers at dosages of $5,7.5,10$ and $15 \mathrm{~kg}$ per $\mathrm{m}^{3}$ of concrete.

- Fibre-reinforced shotcrete using macro steel fibres of various types and suppliers at dosages of $25,30,40$ and $80 \mathrm{~kg}$ per $\mathrm{m}^{3}$ of concrete.

- Various combinations of shotcrete with mesh mounted on either the downside or the outside of the round panel or casted within the concrete.

- Various combinations of shotcrete with mesh mounted on either the downside or the outside of the round panel or casted within the concrete, some including fibres (either macro steel or polypropylene macro fibres).

- Mesh only.

In order to provide good comparability of data when it comes to concrete panels, a key focus was to keep the concrete quality within a narrow range. Only samples that fulfilled a $C 40 / 50$ concrete with a water binder ratio between 0.46 and 0.48 were accepted. Different types of steel fibres were used, including from the following suppliers: HIC, Baekert and Kasturi. Macro synthetic fibres were used from Sika (SikaFibre 60) and Barchip (Shogun 54). All panel data were obtained after a panel curing time of 28 days at room temperature. One large testing series focused on the early-age strength gain of fibre-reinforced panels in the first 36 hours of curing. Both polypropylene macro fibres and macro steel fibres were used for this test series. Figure 2 displays the ASTM panel results for fibre-reinforced ASTM panels with some also including a welded wire mesh. The fibre dosage is plotted along the $X$-axis, and the absorbed energy after $40 \mathrm{~mm}$ of deflection is plotted on the $\mathrm{Y}$-axis. Highest energy absorptions with an in-cycle fibre-reinforced shotcrete can be reached by dosing good-quality polypropylene macro fibre at a dosage of $8-10 \mathrm{~kg}$. This dosage is seen to remain manageable in large-volume shotcrete production without causing major issues during the concrete production and spraying process. The workability limits for both the polypropylene macro fibres and the steel fibres are indicated below the $\mathrm{X}$-axis in Figure 2.

To achieve similar energy absorptions using the same concrete, a far higher dosage of steel fibres is needed. However, it is not possible to match the same energy absorption levels with steel fibres, even at a high dosage of $40 \mathrm{~kg}$ per $\mathrm{m}^{3}$ shotcrete. Note in Figure 2 that the higher value for steel fibres at around 1,100 joules at a $40 \mathrm{~kg}$ dosage is for a materially thicker panel $(100 \mathrm{~mm})$ than specified in the ASTM testing protocol, which is a panel thickness of only $75 \mathrm{~mm}$. Dosages of around $25 \mathrm{~kg}$ of steel fibres result in overall low energy absorption values, as shown in Figure 2. A combination of steel and polypropylene macro synthetic fibres has not resulted in a materially higher energy absorption compared with a high dosage of high-quality steel fibres alone. Increasing the thickness of the shotcrete liner has a strong effect on the overall energy absorption during the ASTM testing protocol, as indicated in Figure 3.

Moving on from rapid development layouts to the installation of mesh, it is interesting to observe the difference between a well-placed mesh on the outside of the ASTM round panel and a well-placed mesh casted within the round panel. 


\section{$4 \quad$ Results}

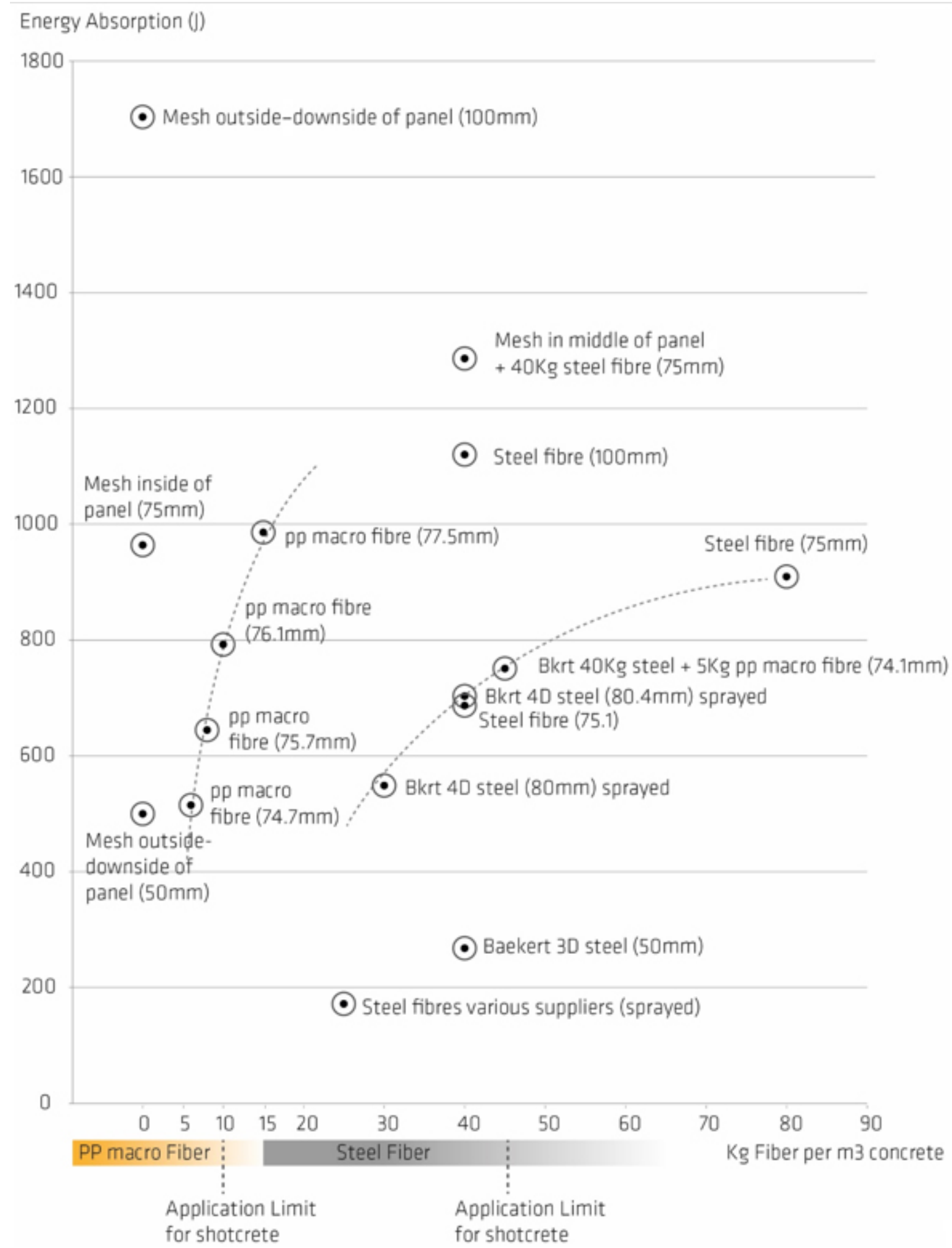

Figure 2 Absorbed energy according to the ASTM C1550 round panel test in relation to the amount of contained fibre or mesh (smooth rod, $8 \mathrm{~mm}$ diameter, $75 \mathrm{~mm}$ spacing). Polypropylene (pp) macro fibre: SikaFibre 60 or Barchip Shogun 54. Steel fibre: Baekert (Bkrt) 3D 65/35 or as stated. Figures in brackets indicate the average panel thickness for each test group. All samples casted unless indicated 


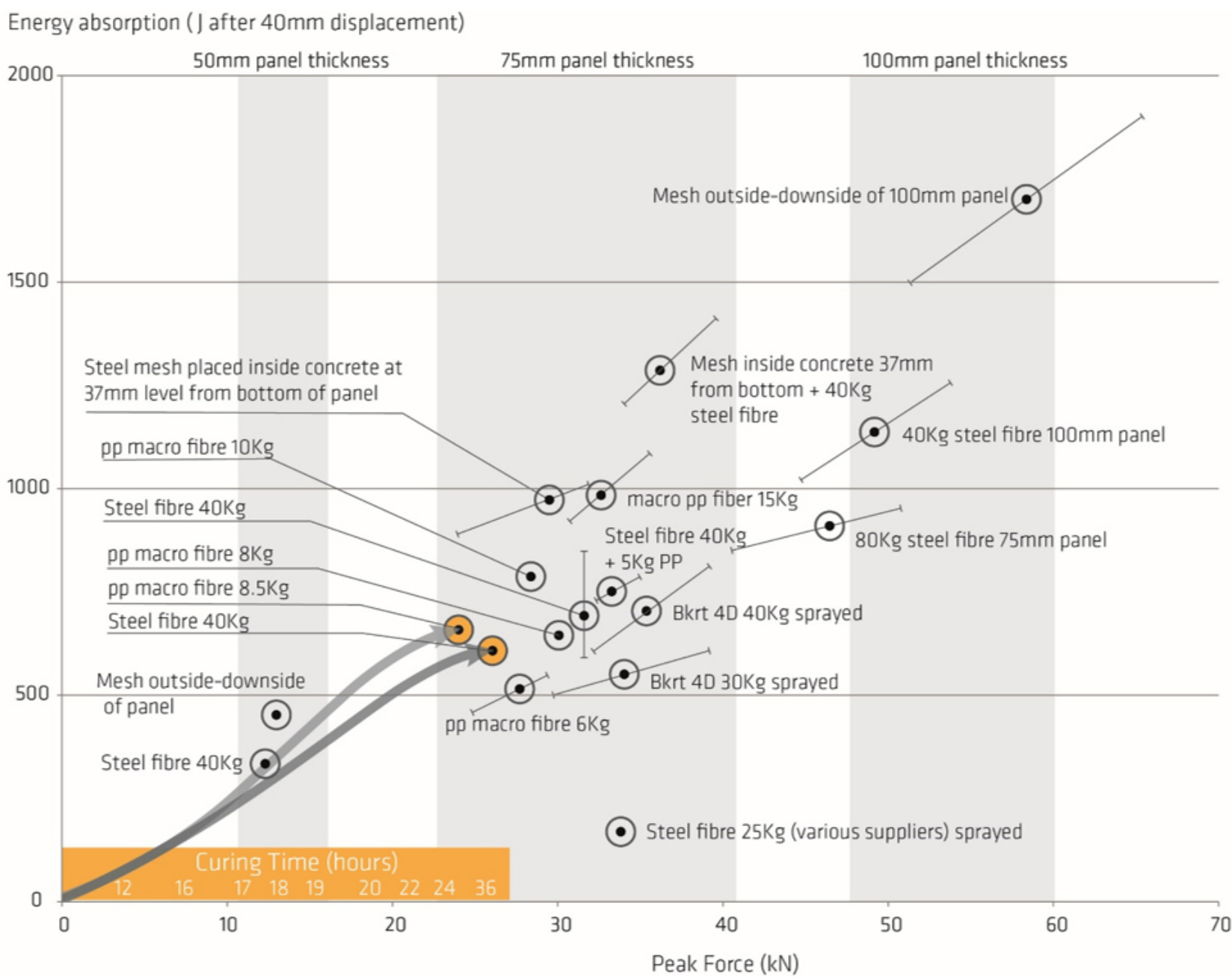

Figure 3 Absorbed energy according to the ASTM C1550 round panel test in relation to the recorded peak force in kN. Mesh: Smooth rod, $8 \mathrm{~mm}$ diameter, $75 \mathrm{~mm}$ spacing. Polypropylene (pp) macro fibre: SikaFibre 60 or Barchip Shogun 54. Steel fibre: Baekert (Bkrt) 3D 65/35 or as stated. Grey arrows display the curing path over the 36 -hour testing interval where steel and pp macro fibres were used. All samples casted unless indicated otherwise

For obvious reasons, the position of the mesh outside or close to the tensile zone within the panel has a favourable effect in terms of energy absorption, as shown in Figure 3. When comparing a steel-meshreinforced $100-\mathrm{mm}$-thick panel with the same set-up but with a $50 \%$ thinner panel, the absorbed energy is reduced by more than $70 \%$. A casted steel mesh, with the mesh located in the middle of the panel, results in an energy absorption that is roughly $15 \%$ higher than a macro-synthetic-fibre-reinforced shotcrete containing around $10 \mathrm{~kg}$ of good-quality macro synthetic fibres. To achieve the same energy absorption, more than $40 \mathrm{~kg}$ of high-quality macro steel fibres would need to be dosed. It is important to watch the peak force closely, which is displayed on the X-axis in Figure 3. This peak force should be in the same range for similar panel thicknesses in order to allow good comparability of data. For the dataset used, this is more or less the case with higher peak forces only occurring when moving to higher panel thicknesses. The peak force indicates the peak loading on the panel once the first crack in the concrete occurs, and a higher peak value usually results in overall better energy absorption due to better embedding of the fibres. Higher peak forces usually mean a better concrete quality. As expected, on a standard 75-mm-thick panel, highest energy absorptions were achieved by combining a mesh- and fibre-reinforced shotcrete. Overall, meshes placed at the outside of the panel resulted in higher overall absorbed energies than meshes placed within the concrete panels. 
Curing of non-accelerated test specimens over a 36-hour period has shown that energy absorption increases significantly over this period, reaching the predicted 28-day value after just 36 hours as shown in Figure 3 where the curing time and strength build-up is displayed for two panel populations: one containing steel fibres, the other polypropylene macro fibres. Curing took place at room temperature. Looking at the absorbed energy over the deflection span, a casted/sprayed mesh in combination with fibres results in a homogeneous energy absorption over the full length of the deflection curve. This can be seen in Figure 4 .

Loading Force on panel $(\mathrm{kN})$

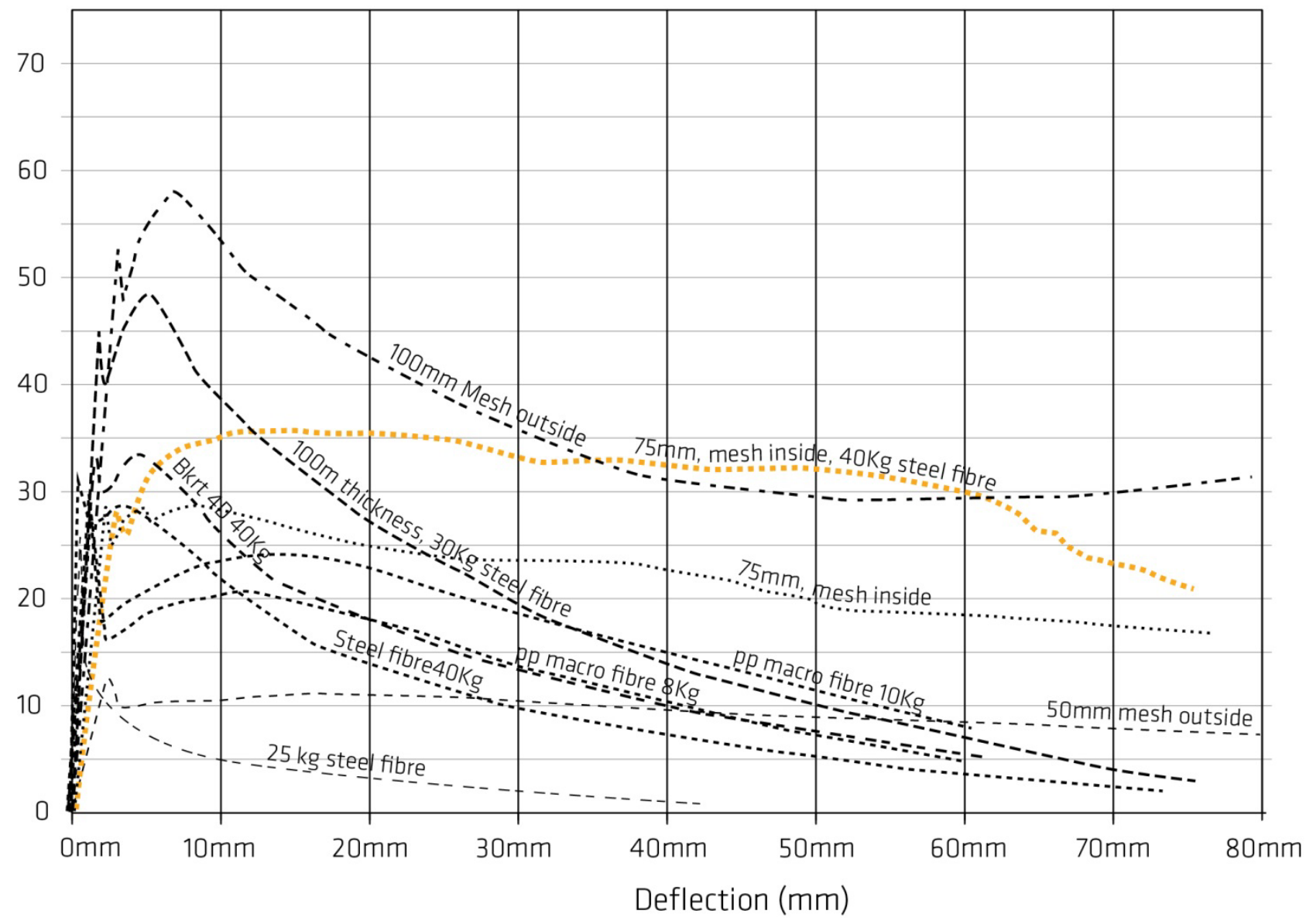

Figure 4 Averaged results of absorbed forces during ASTM C1550 deflection test. Note that the curves with the casted mesh inside the panel result in a continuous intake of energy over the deflection curve. Mesh: Smooth rod, $8 \mathrm{~mm}$ diameter, $75 \mathrm{~mm}$ spacing. Polypropylene (pp) macro fibre: SikaFibre 60 or Barchip Shogun 54. Steel fibre: Baekert (Bkrt) 3D 65/35. All samples casted using standard ASTM round panels

Results from dynamic impact testing showed that liner thickness has a large impact on the absorbed energy of reinforced shotcrete, not only on the overall absorbed energy but also on the deflection length during impact. This can be seen in Figure 5, where the original averaged data for each test series is displayed.

Deflection of the panel during impact is greatly reduced with increased liner thicknesses. Deflection decreases by more than 70\%, going from $50 \mathrm{~mm}$ fibre-reinforced liner thickness to $100 \mathrm{~mm}$ liner thickness, all in combination with a mesh mounted on the lower side of the panel. By combining a well-placed mesh with fibre-reinforced concrete, energy loading as well as dynamic energy impact can be improved by close to $70 \%$ compared with a dosage of $10 \mathrm{~kg}$ of a high-quality polypropylene fibre or $40 \mathrm{~kg}$ of steel fibres without mesh. 
Note that the overall limitation of a pure fibre-reinforced shotcrete is clearly defined by the degree of deflection. Once the fibre length is pulled out, nothing can prevent the shotcrete liner from failing. This critical deflection is reached at around $50 \mathrm{~mm}$ for steel fibres and $75 \mathrm{~mm}$ for macro synthetic fibres. Above these deflection values, all fibre-reinforced panels fail. It is interesting that when increasing the panel thickness from $75 \mathrm{~mm}$ to $100 \mathrm{~mm}$ the increase of energy absorption shifts from around 1,250 to 2,000 joules, matching energy absorption values of a support system that includes the same amount of steel fibres in combination with a wire mesh for the same drop height and weight of projectile.

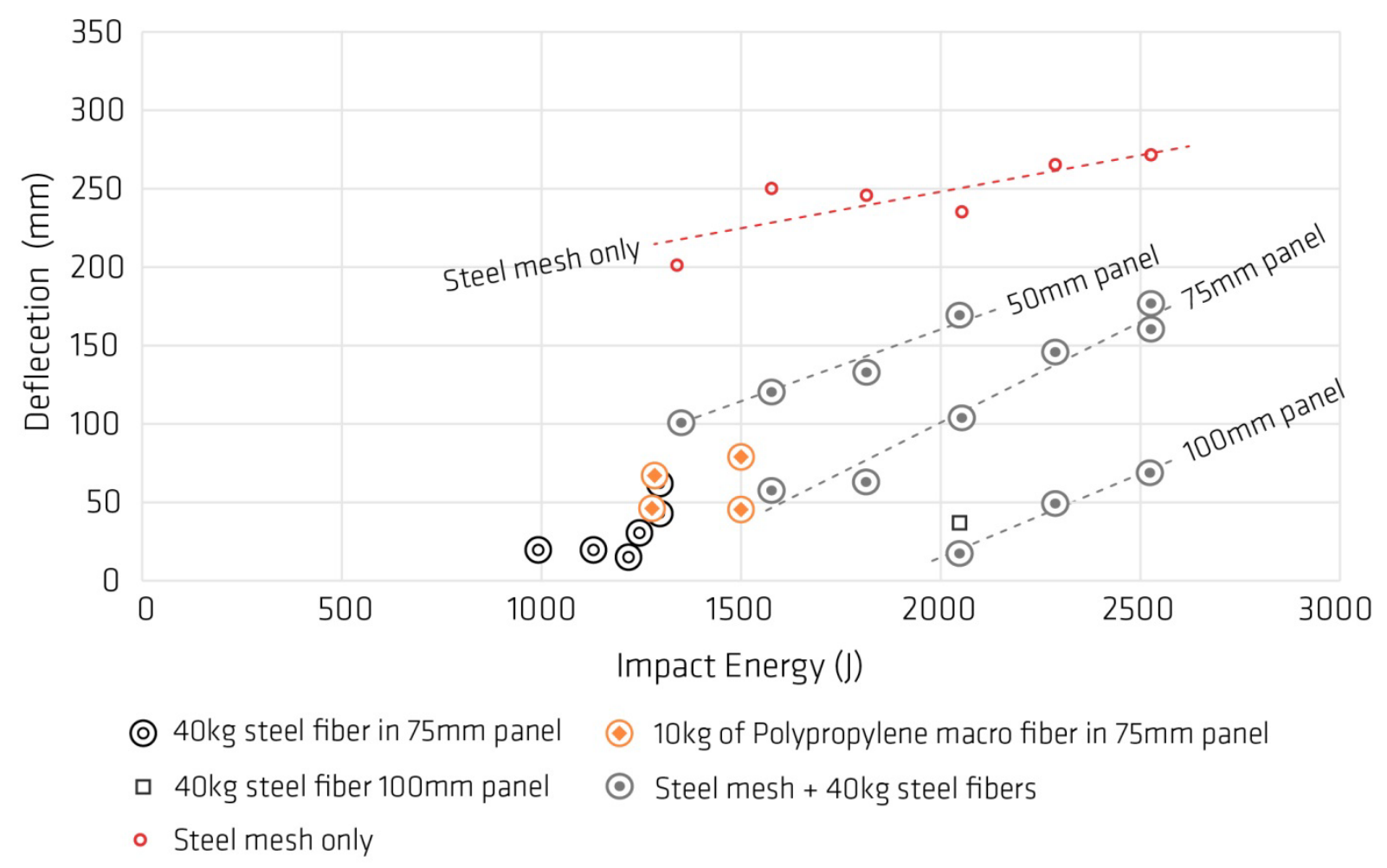

Figure 5 Absorbed impact energy calculated based on the drop height and weight of the used projectile in relation to the resulted deflection during the impact. Mesh: Smooth rod, $8 \mathrm{~mm}$ diameter, $75 \mathrm{~mm}$ spacing. Polypropylene macro fibre: SikaFibre 60 or Barchip Shogun 54. Steel fibre: Baekert 3D 65/35. All samples casted using standard ASTM round panels. Modified from Swedberg \& Krutrök (2016)

\section{Discussion}

Testing according to the ASTM C1550 protocol and combining the results with dynamic impact testing results showed that high energy absorption and high impact energies could be best countered by combining a good-quality fibre-reinforced shotcrete with a well-placed mesh, either outside the shotcrete liner or within the liner. Despite the favourable behaviour of a sprayed-in mesh under constant loading, where the support system takes on loading very homogeneously over the deflection curve, a few points need to be considered. Shotcrete over a mesh results in higher rebound and spray shadows behind the mesh. The process within an in-cycle set-up is time consuming and costly and requires multiple installation processes that will eventually include work under unsupported roof and rib sections. 
The installation of the mesh at the outside of the sprayed concrete, where needed, appears to be the more cost efficient and better option from a practical point of view. This will also mitigate the risk of corrosion inside the liner, and the quality and integrity of the placed mesh can be observed over time. It also needs to be emphasised that the location of the placed mesh within the shotcrete liner (if sprayed or casted) is critical to the overall energy absorption. In reality, the placement is very often unfavourable because the mesh is bolted relatively tightly to the rock surface and hence is not positioned in the tensile zone of the subsequently sprayed concrete. This is cause for major spalling of sprayed unreinforced concrete on top of the placed mesh during rapid deformational events. Mesh installations are particularly effective at large deflection rates where fibre pull-out would result in a failure of the system, as the fibres will reach the capacity limits at roughly a crack opening width that corresponds to half the fibre length. In terms of used fibres, the test results showed the superior performance of macro polypropylene fibres compared with good-quality steel fibres, especially when it comes to dynamic loading of the panels during sudden seismic shocks and destressing events. Macro synthetic fibres have shown to take on significantly more deflection during fast impact loading. Increasing the overall shotcrete thickness has shown to increase the energy absorption substantially, to a point where the difference between a wire-mesh-reinforced panel combined with fibres compared with a purely fibre-reinforced shotcrete is significantly reduced, as shown in Figure 5. Considering the better yielding capabilities of polypropylene macro fibres compared with steel fibres at much lower dosage as well as the non-corrosive nature of polypropylene material, this also clearly speaks for the use of macro synthetic fibres from a cost-performance and risk point of view. Active corrosion within or behind the shotcrete liner is the great unknown in mining environments where acid drainage waters from the sulphide-bearing host rock has a detrimental effect on the long-term integrity of any steel-reinforced support. Polypropylene macro fibres will have a positive impact on the long-term integrity of the shotcrete liner and will form a last line of defence in a corrosive environment that will impact any steel component of a sprayed concrete support, as shown by Kaufmann \& Manser (2013) or Bernard (2004), where shotcrete panels reinforced with steel and polypropylene macro fibres were exposed to a corrosive environment and retested after one year of exposure.

A combination of fibres and mesh has been proven very favourable in terms of energy absorption not only over limited deflection spans but also for large energy absorption over wider deflection during dynamic impact testing. By choosing such a combination, two main advantages can be realised:

1. The initial stiffness and yielding capabilities of the fibre-reinforced shotcrete.

2. An excellent yielding element from the mesh over larger, but also reduced, deflection spans when compared with pure mesh and bolt installations.

Test results from early-stage curing of shotcrete liners proved that early strength development plays a key role when it comes to the active support that the shotcrete liner provides to the underlying strata. When considering rapid mine development projects, the strength build-up of the sprayed concrete takes a considerable amount of time during a blast cycle. Over the years, several rules of thumb have been established in the industry. One has been that re-entry into a sprayed perimeter is not safe before a uniaxial compressive strength (UCS) of $1 \mathrm{MPa}$ has been reached, because the liner would not be capable of holding up its own weight and especially not capable of providing active support to the underlying strata. 
Extensive field trials from Bernard (2009), correlating UCS values with actual shear strength values of fibre-reinforced shotcrete showed that fibres start to take on load significantly earlier and that a reinforced liner is well capable of supporting its own and additional weight earlier than the $1 \mathrm{MPa}$ hurdle. In addition to Bernard's findings regarding very early strength development, it could be extrapolated from this study that early yielding of the liner builds up rapidly within the first 36 hours and achieves almost the entire design capacity after this time, as shown in Figure 3.

\section{Outlook}

Due to the importance of early strength build-up in the shotcrete liner (Erismann et al. 2018), Sika developed a tool to measure early strength development within the shotcrete liner with an in situ portable ultrasonic pulsation device. This tool is a further development of the existing Sika MiniShot ${ }^{\circledR}$ device that uses the same system as part of a laboratory-based shotcrete evaluation and quality control tool, as described by Lindlar et al. (2014), Lootens et al. (2014), and Oblak et al. (2012). This tool is considered groundbreaking in its effect on the evaluation of safe re-entry times of freshly sprayed perimeters, and it will enable quality control of large-scale shotcrete applications at a much improved and reliable level. As shown in Figure 6, the portable strength measurement device is pushed into the freshly sprayed shotcrete substrate (Figure 6a), and once the ultrasonic sensor is in contact with the concrete surface, it starts to record real-time hardening of the sprayed concrete. The ultrasonic waves are reflected by the substrate, captured by the receiver and then translated into a strength signal using a dedicated algorithm.

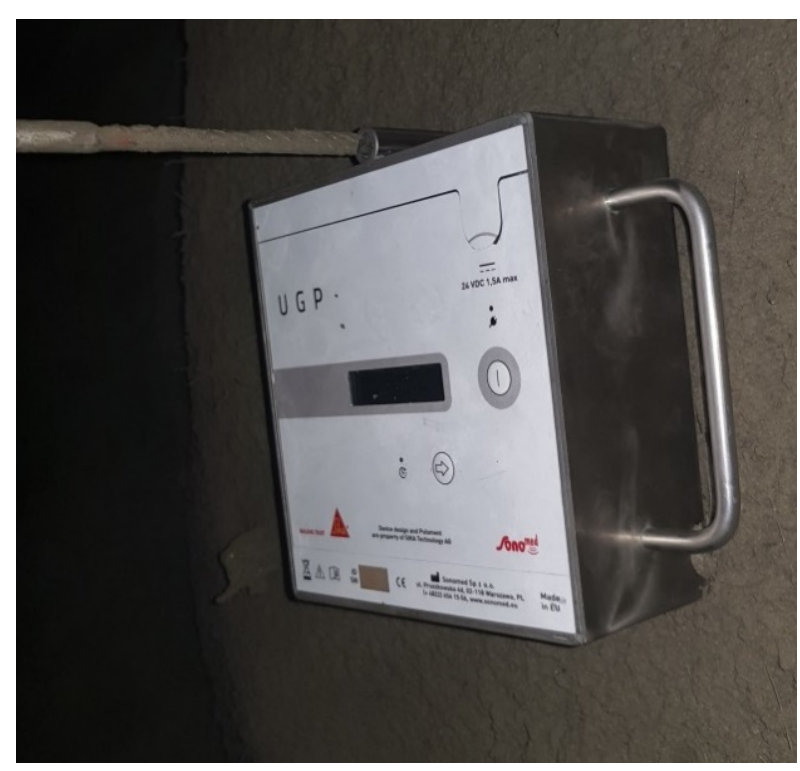

(a)

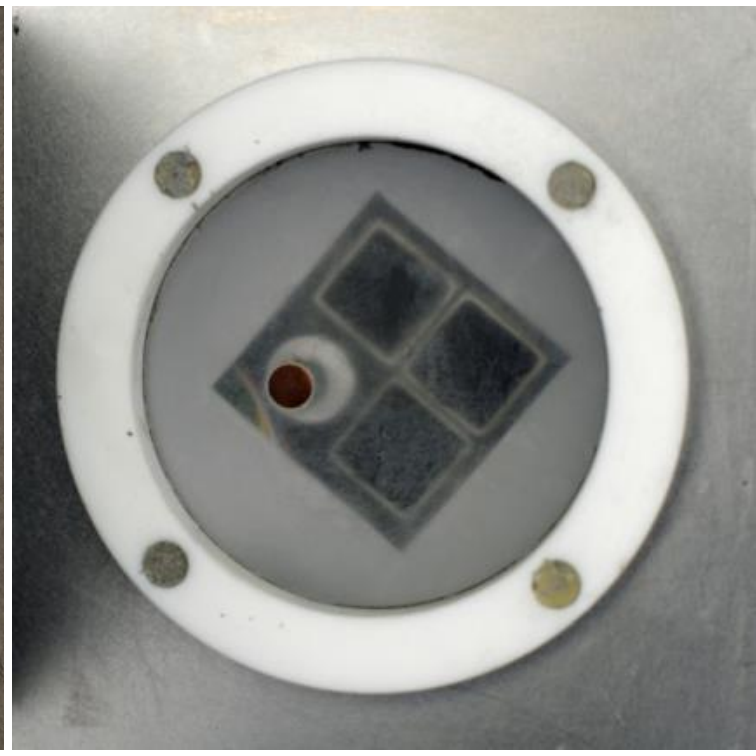

(b)

Figure 6 (a) Onsite in situ measurement tool; (b) Based on an ultrasonic receiver. The tool is pressed into the freshly sprayed concrete, measures the strength build-up immediately and then transfers the data live via a Wi-Fi signal

The result of the measurement is a real-time strength development curve of the sprayed concrete, as shown in Figure 7b. Figure 7 displays results from the ultrasonic device, where strength development has been measured. These values were referenced with conventional strength measurements of the same shotcrete. A penetrometer for compressive strengths up to $1.5 \mathrm{MPa}$ was used first and then the Hilti method until around $15 \mathrm{MPa}$ was reached, which occurred after approximately 12 hours. Press testing of drilled cylinders was used thereafter. The correlation of the data using the two different strength measurements is remarkable. 


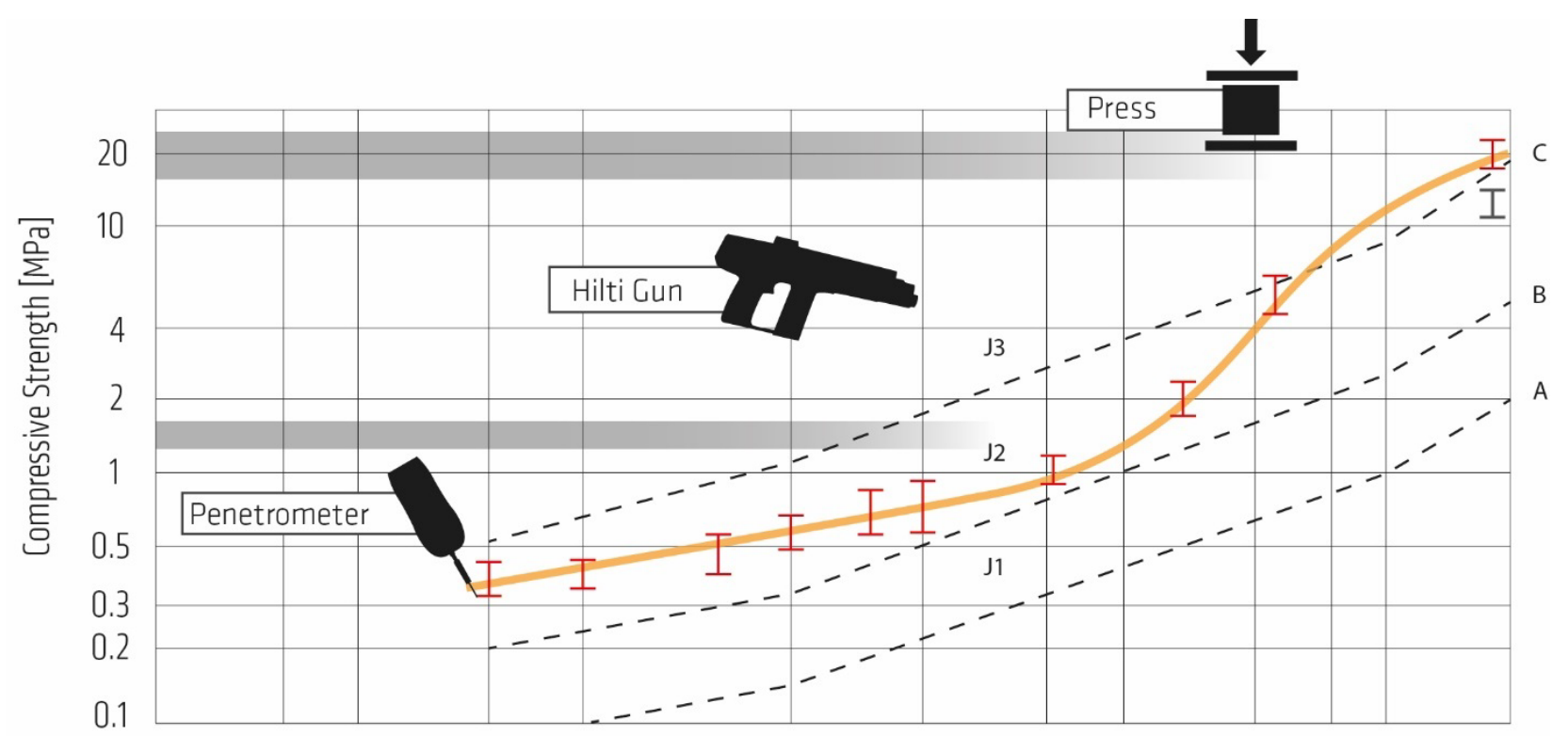

(a)

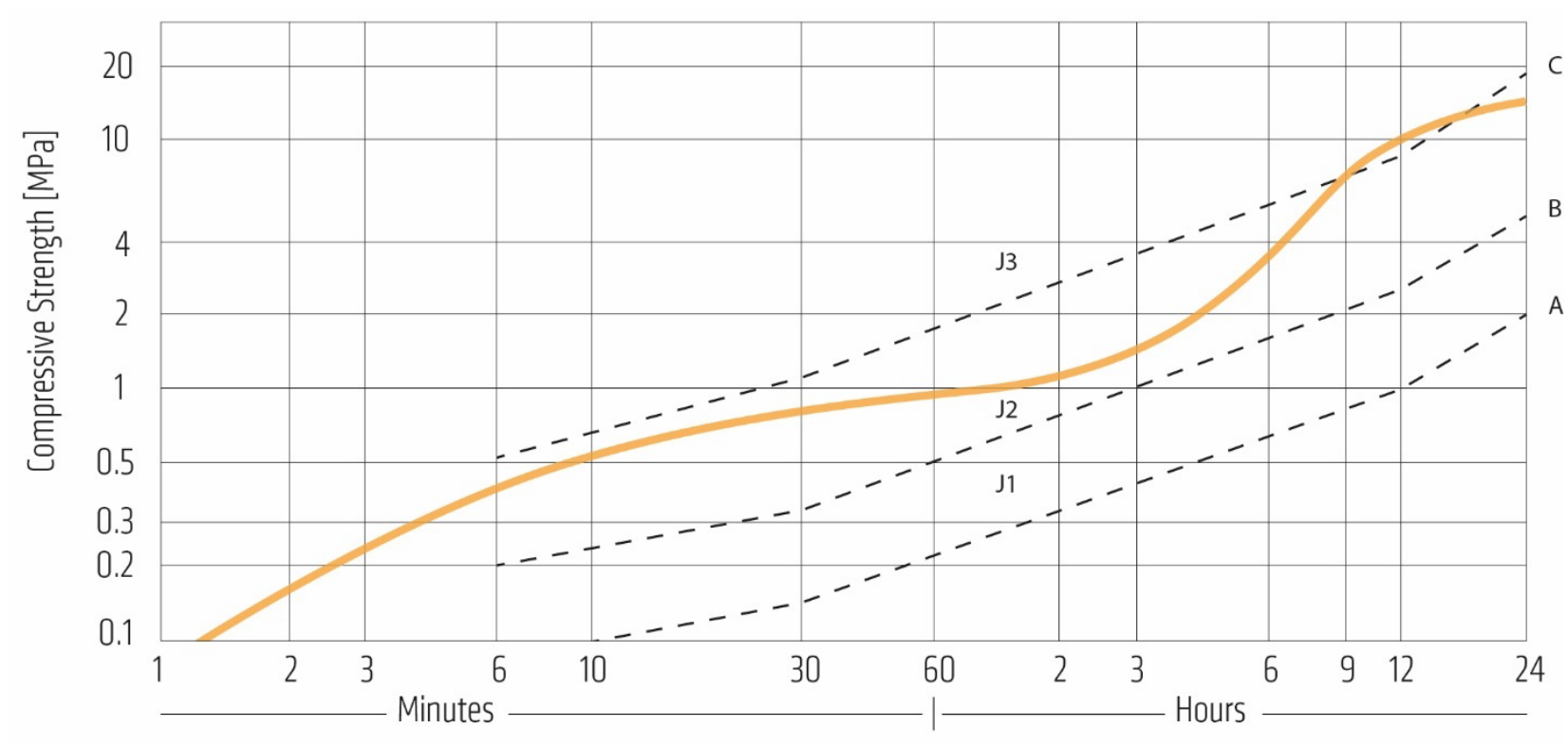

Accelerator A

(b)

Figure 7 (a) Comparison of results of an onsite real-scale shotcrete trial using a penetrometer for early strength, Hilti gun and cylinder press for higher strengths; (b) Measurement with the in situ ultrasonic pulsement tool. There is very good correlation of data between the two

\section{Conclusion}

This study has laid a base for underground support methods that are implemented as best demonstrated practices in several large underground mining projects, including some of the most recent super-cave projects. A fibre-reinforced shotcrete liner is the fastest and most reliable in-cycle support system and has been proven best in class when it comes to the cost performance of the installed primary support. Good yielding at limited deflection, the formation of an almost immediate active support and highly flexible application and scalability are the key success factors of fibre-reinforced shotcrete. When higher deflection rates need to be mitigated and when underground openings are exposed to sudden dynamic loading through destressing events, the combination of fibre-reinforced sprayed concrete with a well-placed mesh 
either inside or outside the liner has proven very effective in also countering larger deformation events and ongoing loading of the liners. Early-age yielding of the sprayed concrete liner reaches designed energy absorption levels much earlier than is commonly accepted within the industry. This study has shown that design strength can be reached as early as 36 hours. Therefore, the common practice of testing shotcrete panels after 28 days needs reconsideration. Onsite in situ tools for measuring strength build-up of sprayed shotcrete liners will be of interest for further increasing blast cycle times and speeding up safe and early re-entry into freshly sprayed perimeters.

\section{Acknowledgement}

The authors thank LKAB for publishing this data and, in particular, Erik Swedberg and Benjamin Krutrök for their ongoing support and collaboration.

\section{References}

Amberg, F 2006, Tunnelling in High Overburden: The Swiss Approach, ITA/AITES Training Course, Tunnel Engineering, Seoul. ASTM International 2012, Standard Test Method for Flexural Toughness of Fibre Reinforced Concrete (Using Centrally Loaded Round Panel) (ASTM C1550-12a), ASTM International, West Conshohocken.

Bernard, ES 2004 (ed.), 'Durability of cracked fibre reinforced shotcrete', Shotcrete: More Engineering Developments-Proceedings for the Second International Conference on Engineering Developments in Shotcrete, October 2004, Cairns.

Bernard, ES 2009, 'Early-age re-entry under fresh fibre reinforced shotcrete', Proceedings of Shotcrete for Underground Support XI, Engineering Conferences International, Switzerland.

Erismann, F, Lindlar, B, Munoz, C, Hansson, M \& Erlangga, R 2018, 'Rapid mine development using efficient in-cycle shotcrete', in Y Potvin \& J Jakubec (eds), Proceedings of the Fourth International Symposium on Block and Sublevel Caving, Australian Centre for Geomechanics, Perth, pp. 759-766.

Hadjigeorgiou, J \& Potvin, Y 2011, 'A critical assessment of dynamic rock reinforcement and support testing facilities', Rock Mechanics and Rock Engineering, vol. 44, pp. 565-578.

Kaufmann, J \& Manser, M 2013, 'Durability performance of bi-component polymer fibres under creep and in aggressive environments', in B Brady \& Y Potvin (eds), Proceedings of the Seventh International Symposium on Ground Support in Mining and Underground Construction, Australian Centre for Geomechanics, Perth, pp. 585-596.

Lindlar, B, Oblak, L, Lootens, D \& Stenger, C 2014, 'From tunnel to laboratory: Scaling of shotcrete testing', paper presented at the 7th International Symposium on Sprayed Concrete, Sandefjord, 16-19 June.

Lootens, D, Hansson, M, Oblak, L, Lindlar, B 2014, 'Ultrasonic wave propagation for strength measurements: Application in Shotcrete', paper presented at the 7th International Symposium on Sprayed Concrete, Sandefjord, 16-19 June.

Oblak, L, Lindlar, B, Lootens, D 2012, 'Continuous monitoring of strength evolution of shotcrete', paper presented at the 10th International Shotcrete Conference, Alpbach, 12-13 January.

Swedberg, E 2013, Tidig Hållfasthet för Sprutbetong och Cementingjuten Bergbult, PhD thesis, Luleå University of Technology, Luleå.

Swedburg, E, Thyni, F, Töyrä, J \& Eitzenberger, A 2014, 'Rock support testing in Luossavaara-Kiirunavaara AB's underground mines, Sweden', in M Hudyma \& Y Potvin (eds), Proceedings of the Seventh International Conference on Deep and High Stress Mining, Australian Centre for Geomechanics, Perth, pp. 139-150.

Swedberg, E \& Krutrök, B 2016, 'Drop testing of concrete panels and welded wire mesh at LKABs Kiirunavaara mine', in E Nordlund, TH Jones \& A Eitzenberger (eds), Proceedings of the Eighth International Symposium on Ground Support in Mining and Underground Construction, Luleå University of Technology, Luleå.

Thyni, F 2014, Design of Shotcrete for Dynamic Rock Support by Static Testing, PhD thesis, Luleå University of Technology, Luleå. 
Early strength development of shotcrete for rapid mine

F Erismann \& M Hansson development and behaviour under dynamic loads 